\title{
ON FUNCTIONAL IDENTITIES OF DEGREE 2 AND CENTRALIZING MAPS IN TRIANGULAR RINGS
}

\author{
YU WANG
}

Abstract. Let $R$ be a triangular ring with center $Z(R)$. Let $F_{1}, F_{2}, G_{1}, G_{2}: R \rightarrow R$ be maps such that

$$
F_{1}(x) y+F_{2}(y) x+x G_{2}(y)+y G_{1}(x) \in Z(R)
$$

for all $x, y \in R$. The aim of the paper is to give a solution of this functional identity in certain triangular rings. As applications, centralizing additive maps of certain triangular rings are determined.

Mathematics subject classification (2010): 16R60, 16S50, 47L35.

Keywords and phrases: Functional identity, triangular ring, upper triangular matrix ring, nest algebra, centralizing additive map.

\section{REFERENCES}

[1] D. Benkovič, D. EREMITA, Commuting traces and commutativity preserving maps on triangular algebras, J. Algebra 280 (2004), 797-824.

[2] M. BREŠAR, Centralizing mappings and derivations in prime rings, J. Algebra 156 (1993), $385-94$.

[3] M. BREŠ AR, On generalized biderivations and related maps, J. Algebra 172 (1995), 764-786.

[4] M. BREŠ AR, Commuting maps: a survey, Taiwanese J. Math. 8 (2004), 361-397.

[5] M. Brešar, M. A. Chebotar, W. S. Martindale 3RD, Functional identities, Frontiers in mathematics, Basel: Birkhäuser, 2007.

[6] W.-S. Cheung, Commuting maps of triangular algebras, J. London Math. Soc. 63 (2001) 117-127.

[7] K. R. Davidson, Nest Algebras, in: Pitman Res. Notes Math. Ser., vol. 191, Longmans, Harlow, 1988.

[8] Y. Q. DU, Y. WANG, k-Commuting maps on triangular algebras, Linear Algebra Appl. 436 (2012) 1367-1375.

[9] D. EREMITA, Functional identities of degree 2 in triangular rings, Linear Algebra Appl. 438 (2013) 584-597.

[10] D. EREMitA, Functional identities of degree 2 in triangular rings revisited, Linear and Multilinear Algebra 63 (2015) 534-553.

[11] E. C. Posner, Derivations in prime rings, Proc. Amer. Math. Soc. 8 (1957) 1093-1100.

[12] Y. WANG, Functional identities of degree 2 in arbitrary triangular rings, Linear Algebra Appl. 479 (2015) 171-184.

[13] Y. WANG, Commuting (centralizing) traces and Lie (triple) isomorphisms on triangular algebras revisited, Linear Algebra Appl. 488 (2016) 45-70.

[14] J. H. Zhang, S. FEnG, H. X. LI, R. H. Wu, Generalized biderivations of nest algebras, Linear Algebra Appl. 418 (2006) 225-233. 